\title{
Effect of pimobendan on left atrial function in dogs with preclinical myxomatous mitral valve disease
}

\author{
Fabio Sarcinella*, Joao Neves, Thomas W. Maddox, Hannah M. Hodgkiss-Geere, Elizabeth F. Bode and \\ Joanna Dukes-McEwan \\ Department of Small Animal Clinical Science, Small Animal Teaching Hospital, Institute of Veterinary Science, \\ University of Liverpool, Neston, Chester CH64 7TE, UK
}

\begin{abstract}
Background: Left atrial (LA) function is an important determinant of the left ventricular (LV) filling, playing a key role in maintaining optimal cardiac performance. Pimobendan is a phosphodiesterase III inhibitor with positive inotropic and vasodilator effects. The present study aims to investigate the effects of pimobendan on LA function in dogs with stage B2 myxomatous mitral valve disease (MMVD).

Aim: The aim of this investigation was to study the effects of pimobendan on LA function in dogs with preclinical MMVD.

Methods: Twenty-seven dogs with stage B2 MMVD were retrospectively included. LA function was assessed before and 1-6 months following pimobendan initiation. For each dog, two-dimensional (2D) echocardiography was performed to assess LA diameter and volume for each phase of the LA cycle and to assess complete, passive, and active LA function. Pulsed-wave tissue Doppler imaging (TDI) of the left ventricular longitudinal myocardial velocity associated with atrial contraction(A'), bothattheleveloftheinterventricularseptumandtheLV freewall, wasalsousedasanindicatorofLAfunction. Results: There were no significant differences in any of the left atrial variables pre- and posttreatment.

Conclusion: Echocardiographic estimates of LA function by 2D diameters and volumes and TDI A' in dogs with MMVD do not change after treatment with pimobendan.

Keywords: Atrioventricular valve degeneration, Canine, Chronic valvular disease, Phosphodiesterase inhibitor, Vetmedin.
\end{abstract}

\section{Introduction}

Myxomatous mitral valve disease (MMVD) is the most prevalent cardiac disease in dogs and is characterized by a slow, progressive myxomatous degeneration of the mitral valve leaflets, leading to mitral valve regurgitation (MR) of increasing severity and subsequent left atrial (LA) and left ventricular (LV) dilatation (Ljungvall and Häggström, 2016). In particular, LA remodeling and enlargement can be observed during early stages of the disease (Ljungvall and Häggström, 2016) reflecting LA damage by MR. The LA modulates LV filling through three major functions in sinus rhythm: reservoir, during ventricular systole; conduit, during early ventricular diastole; and pump (active contractile function), during late ventricular diastole. Throughout atrial contraction, the LA contributes to $15 \%-30 \%$ of LV filling (Tidholm et al., 2013). However, the LA contribution to LV filling in patients with congestive heart failure (CHF) gradually decreases with progression of LV dysfunction (Nakamura et al., 2014). In humans with MR, LA dysfunction has been reported to correlate with disease severity and cardiovascular events (Benjamin et al., 1995; Pritchett et al., 2003). Similarly, in dogs, echocardiographic variables of LA dimension, morphology, and function have been reported to be strong predictors of disease severity and cardiac death (Borgarelli et al., 2008; Baron Toaldo et al., 2018). Therefore, the assessment of LA function can provide important insights about pathophysiology and prognosis in people and dogs with MR (Benjamin et al., 1995; Pritchett et al., 2003; Nakamura et al., 2014). Both in human and veterinary medicine, several methods for the assessment of LA function have been used, including 2D echocardiography (echo), tissue Doppler echo (TDI), three-dimensional echo, and magnetic resonance imaging (Hauser et al., 2008; Baron Toaldo et al., 2014; Fries et al., 2019). However, at present, 2D echo remains the simplest, least invasive, and most cost-effective method for such evaluation (Blume et al., 2011; Roşca et al., 2011).

Pimobendan is an inodilator, exerting its inotropic effect by a dual mechanism of action consisting of calcium sensitization of the cardiac troponin $\mathrm{C}$ molecule and inhibition of phosphodiesterase III (Boyle and Leech, 2012). This drug has been proven to be safe and effective for the treatment of CHF secondary to MMVD to decrease overall heart size in dogs with MMVD and experimental MR (Suzuki et al., 2011; Boswood et al., 2016) and to significantly reduce LA pressure in dogs with surgically induced MR (Suzuki et al., 2011). Pimobendan has also been shown to have beneficial effects in postponing the onset of left-sided CHF 
and cardiac death in subclinically affected dogs with cardiomegaly due to MMVD (Boswood et al., 2016). Although it is known to reduce LV diameters in systole and diastole and improve systolic and diastolic function (Suzuki et al., 2011; Boswood et al., 2016), the effect of pimobendan on LA mechanical phasic function in dogs affected by MMVD has not been studied despite the well-recognized importance of the contribution of atrial contraction to ventricular filling (Nakamura et al., 2014). Furthermore, conflicting results on the effect of this drug on cats' LA function have been reported (Kent, 2011; Oldach et al., 2019). The aim of this investigation was to study the effects of pimobendan on LA function in dogs with preclinical MMVD. On the basis of the known actions of pimobendan, we hypothesized that oral administration of pimobendan in dogs with stage B2 MMVD, not receiving any other cardiovascular medications, would cause a reduction in LA size and an improvement in LA active function.

\section{Materials and Methods}

This was a retrospective study. The medical records of all canine patients referred to the cardiology service of the Small Animal Teaching Hospital, University of Liverpool, for investigations of a heart murmur, between 2015 and 2018, were reviewed for suitability for inclusion in this study. Dogs that had undergone echo and were coded as stage B2 and MMVD in the EchoPac (EchoPac, General Electric Medical System, Buckinghamshire, UK) analysis system were eligible for inclusion, providing that they were prescribed pimobendan treatment, based on the echocardiographic criteria proposed by the EPIC study (short-axis early diastolic left atrial-to-aortic diameter ratio (LA/Ao) $\geq 1.6$ and $\mathrm{M}$-mode left ventricular internal diameter normalized for body weight (LVIDDN) $\geq 1.7$ ) (Hansson et al., 2002; Cornell et al., 2004; Boswood et al., 2016), and that a repeat echocardiographic study was performed within 6 months from initiation of pimobendan. The exclusion criteria included the presence of any evidence of cardiovascular disease (other than stage B2 MMVD), clinical signs of CHF or systemic disease expected to affect cardiovascular function, the use of any cardiac medications before the first echo, body weight over $30 \mathrm{~kg}$, and lack of followup echo within the 6 months after starting pimobendan. Doppler echo was carried out by board-certified cardiologists or supervised residents with a GE Vivid 7 (Vivid 7, General Electric Medical System, Buckinghamshire, UK) ultrasound machine equipped with 7- and 4-MHz transducers and simultaneous single-lead electrocardiogram. Echocardiography was performed unsedated, with dogs gently restrained in lateral recumbency. The standard echocardiographic views were acquired (Thomas et al., 1993) and measurement protocols were followed at the time of the assessment. The endocardial-blood pool interface defined the measurement boundaries for $2 \mathrm{D}$ echo.
M-mode measurements used the leading edge-leading edge methodology (Sahn et al., 1978). Color flow, pulsed-wave, and continuous-wave Doppler were used to document intracardiac flow velocities, laminar or turbulent flow, and presence of valvular regurgitation. The pulsed-wave (PW) TDI studies from the left apical four-chamber view recordings, parallel to each wall, included basal interventricular septal and left ventricular free wall S', E', and A' velocities. Diagnostic criteria for MMVD included echocardiographic identification of thickened and/or prolapsed mitral valve leaflets and the presence of MR (Boswood et al., 2016).

All the echocardiographic measurements were subsequently performed offline by one author (FS) on a standalone offline measuring system ${ }^{\mathrm{a}}$. The mean value of three, not necessarily consecutive, cardiac cycles in sinus rhythm for each variable was obtained and used for the analysis. For the purpose of this study, a particular attention was paid to the assessment of LA diameter, volume, and function. From stored cine-loops, the following measurements were made: left atrial diameter (LADmax) was obtained from the right parasternal (RPS) long-axis four-chamber view, from interatrial septum to LA lateral wall, as previously described (Rishniw and Erb, 2000); the aortic annulus systolic diameter was measured between the hinge-points of open aortic valves from a RPS five-chamber view, optimizing the aorta; and the systolic LADmax/Ao ratio was calculated (Strohm et al., 2018). The short-axis left atrial-to-aortic ratio (LA/ Ao) was measured in early diastole from the RPS shortaxis view, optimizing LA size (Hansson et al., 2002).

For the assessment of LA volumes (LAV), from the RPS long-axis four-chambered view, optimizing LA size, the single-plane Simpson's formula was used (Russo et al., 2010; Linney et al., 2014). Each variable of LA linear dimensions and volumes was calculated at specified time points throughout each phase of the LA function cycle: at ventricular endsystole (LADmax and LAVmax), one frame before opening of the mitral valve - immediately before the $\mathrm{P}$ wave of the electrocardiographic trace (LADmid and LAVmid), and at ventricular end-diastole (LADmin and LAVmin), one frame before closure of the mitral valve. These variables were used to assess complete, passive, and active LA function, using the formulas reported in Table 1 and as previously described (Linney et al., 2014). Changes in diameter were expressed as fractional shortening (LA-FS) and changes in LAV were expressed as ejection fraction (LA-EF).

Pulsed-wave (PW) TDI of the left ventricular longitudinal myocardial velocities associated with atrial contraction $\left(\mathrm{A}^{\prime}\right)$, both from the interventricular septum and the LV free wall, was measured as additional markers of LA active function (Blume et al., 2011). Mean atrial systolic mitral annular velocity (Ann-A') was also calculated averaging the data obtained by PWTDI of the interventricular septum and LV free wall, as previously performed in people (Hesse et al., 2004). 
Table 1. Calculation method of the left atrial emptying function.

\begin{tabular}{lll}
\hline $\begin{array}{l}\text { Passive empting function } \\
\text { (diameters)-fractional shortening }\end{array}$ & PassEmpD-FS & (LADmax-LADmid)/LADmax \\
$\begin{array}{l}\text { Active empting function } \\
\text { (diameters) - fractional shortening }\end{array}$ & ActEmpD-FS & (LADmid-LADmin)/LADmid \\
\hline $\begin{array}{l}\text { Complete empting function } \\
\text { (diameters)-fractional shortening }\end{array}$ & CompEmpD-FS & (LADmax-LADmin)/LADmax \\
$\begin{array}{l}\text { Passive empting function } \\
\text { (volumes) - ejection fraction }\end{array}$ & PassEmpV-EF & (LAVmax-LAVmid)/LAVmax \\
$\begin{array}{l}\text { Active empting function } \\
\text { (volumes)-ejection fraction }\end{array}$ & ActEmpV-EF & (LAVmid-LAVmin)/LAVmid \\
$\begin{array}{l}\text { Complete empting function } \\
\text { (volumes)-ejection fraction }\end{array}$ & ComEmpV-EF & (LAVmax-LAVmin)/LAVmax \\
\hline
\end{tabular}

(LA): left atrium; (LAD): left atrial diameter; (LAV): left atrial volume; (FS): fractional shortening; (EF): ejection fraction; (LADmax): left atrial linear dimension at the ventricular end-systole; (LADmid): left atrial linear dimension immediately before the P wave on the ECG; (LADmin): left atrial linear dimension at ventricular end-diastole; (LAVmax): left atrial volume at the ventricular end-systole; (LAVmid): left atrial volume immediately before the $\mathrm{P}$ wave on the ECG; (LAVmin): left atrial volume at ventricular end-diastole; (PassEmpD-FS): LA passive function based on diameters; (ActEmpD-FS): LA active function based on diameters; (ComEmpD-FS): LA complete function based on diameters; (PassEmpV-EF): LA passive function based on volumes; (ActEmpV-EF): LA active function based on volumes; (ComEmpV-EF): LA complete function based on volumes. Note: all the FS and EF measurements are expressed as a percentage (formula $\times 100 \%$ ).

For the assessment of LV systolic function, the LV volumes determined by Simpson's method of discs were measured from the RPS long-axis four-chamber view both in diastole and systole as previously described (Wess et al., 2010; 2017). Furthermore, a RPS shortaxis view at the level of the chordae tendineae with the cursor bisecting the LV cavity was obtained to acquire M-mode study of the LV throughout the cardiac cycle as previously reported (Wess et al., 2017). Measurements were then used to calculate Simpson's derived ejection fraction $(\mathrm{EF})$, end-systolic volume index (ESVI-mLs/ $\mathrm{m}^{2}$ ), and M-mode fractional shortening (FS) of the LV, using standard formulas (Wess et al., 2010; 2017) for echocardiographic estimations of LV systolic function. For the time-to-event analysis, a dog was considered to have left-sided CHF when showing clinical signs consistent with left-sided CHF (new onset of tachypnea, dyspnea, and/or exercise intolerance) and/or there was radiographic evidence of cardiogenic pulmonary edema.

\section{Statistical analysis}

All statistical analyses were performed using commercially available statistical software (SPSS 22.0) (SPSS 22.0, SPSS Inc., Chicago, IL). Descriptive statistics were calculated for all variables; continuous data are reported as mean values \pm standard deviation if normally distributed and as median values with interquartile ranges (IQR) if nonnormally distributed. Categorical data are reported as frequencies with $95 \%$ confidence intervals (95\% CI) where appropriate. Normality of distribution for continuous variables was assessed with graphical analysis, probability plots, and the Kolmogorov-Smirnov test. Normally distributed continuous variables were compared before and after treatment using paired $t$-tests, and continuous variables that were not normally distributed were compared using related samples Wilcoxon signed-rank test. For time-to-event analyses, the Kaplan-Meier product limit method was used for the estimation of the median time-to-endpoint and plot time-to-event curves. Dogs without $\mathrm{CHF}$ at the time of final data collection were considered as censored.

\section{Ethical approval}

Ethical approval for this study was granted by the local Veterinary Research Ethics Committee (VREC637).

\section{Results}

\section{Descriptive statistics}

Twenty-seven dogs were included, with a median age of 126 months (IQR 108-140 months) and a median weight of $10.1 \mathrm{~kg}$ (IQR 8.0-10.9 kg). Dog breeds included Cavalier King Charles Spaniels (15), Cocker Spaniels (2), Chihuahuas (2), Miniature Poodles (2), Scottish Terriers (2), crossbreeds (2), Airedale (1), and Miniature Schnauzer (1). Male-neutered dogs were represented by eight dogs, male entire by seven dogs, female neutered by six dogs, and female entire by six dogs. The median time to reexamination following pimobendan administration was 4 months (IQR 3.05.0) with 15/27 dogs undergoing repeat echo between 3 and 4 months (three dogs at $1-2$ months and nine dogs at 5-6 months).

Comparison of variables before and after treatment

All variables except age, time to reexamination, LAVmin, and LAVmid were normally distributed. There were no significant differences in any of the LA variables pre- and posttreatment (Table 2). All the LV systolic function parameters demonstrated a significant 
change $(p<0.05)$ between pre- and posttreatment (Table 2), with increasing FS and Simpson's derived EF ( $p=0.013$ and $p=0.032$, respectively) and decreasing $\operatorname{ESVI}(p=0.003)$.

\section{Time to event}

All dogs but one remained asymptomatic (ACVIM stage B2) during the first 6 months following pimobendan administration. Eleven of the 27 dogs developed CHF over a long-term follow-up with a median time to onset of CHF of 552 days (95\% CI 472.3-631.7 days).

\section{Discussion}

Results of this study indicate that 2D (diameter and volume based) and PW-TDI-derived echo variables of LA size and function are not altered by the administration of pimobendan in dogs affected with stage B2 MMVD, over a period of up to 6 months of treatment administration.

The LA is the main target damaged by chronic MR as this causes increased LA pressure, LA dilation, and degenerative changes. This includes interstitial fibrosis and chronic inflammation (Verheule et al., 2003), leading to reduced LA intrinsic active pump function, which may eventually contribute to the elevation of LA pressure and subsequent development of pulmonary edema. Therefore, preservation of LA function is a potential therapeutic target in dogs affected with MMVD.

Table 2. Echocardiographic variables assessing the left atrial and left ventricular function.

\begin{tabular}{lccc}
\hline Variables & Pretreatment & Posttreatment & p-value \\
\hline TDI lat A' & $10.7 \pm 2.42$ & $11.5 \pm 2.59$ & 0.18 \\
\hline TDI sep A' & $10.2 \pm 3.66$ & $11.1 \pm 3.7$ & 0.2 \\
\hline TDI A'ann & $9.48 \pm 3.56$ & $10.3 \pm 3.38$ & 0.12 \\
\hline MR vel & $5.88 \pm 0.402$ & $6.08 \pm 0.559$ & 0.097 \\
\hline LA/Ao ratio & $1.73 \pm 0.33$ & $1.67 \pm 0.28$ & 0.327 \\
\hline LADmin & $3.07 \pm 0.804$ & $3 \pm 0.791$ & 0.46 \\
\hline LADmid & $3.58 \pm 0.834$ & $3.58 \pm 0.853$ & 0.94 \\
\hline LADmax & $4.04 \pm 0.775$ & $4.02 \pm 0.875$ & 0.74 \\
\hline LAVmin & $14.5 \pm 8.96$ & $14.8 \pm 10.7$ & 0.72 \\
\hline LAVmid & $22.7 \pm 11.6$ & $21.5 \pm 12.6$ & 0.33 \\
\hline LAVmax & $31.6 \pm 14.6$ & $30.8 \pm 16.6$ & 0.6 \\
\hline PasEmpD-FS & $0.118 \pm 0.0745$ & $0.112 \pm 0.0592$ & 0.7 \\
\hline PasEmpV-EF & $0.291 \pm 0.129$ & $0.312 \pm 0.0979$ & 0.48 \\
\hline ActEmpD-FS & $0.148 \pm 0.0733$ & $0.162 \pm 0.0763$ & 0.41 \\
\hline ActEmpV-EF & $0.377 \pm 0.163$ & $0.343 \pm 0.11$ & 0.18 \\
\hline CompEmpD-FS & $0.249 \pm 0.0908$ & $0.256 \pm 0.0784$ & 0.66 \\
\hline CompEmpV-EF & $0.564 \pm 0.128$ & $0.549 \pm 0.0974$ & 0.52 \\
\hline LV-FS & $41.7 \pm 8.93$ & $45.6 \pm 10.6$ & $0.013^{*}$ \\
\hline LV-EF & $67.8 \pm 10.1$ & $71 \pm 10.5$ & $0.032^{*}$ \\
\hline LV-ESVI & $27.8 \pm 12.5$ & $23.8 \pm 11.8$ & $0.003^{*}$ \\
\hline
\end{tabular}

(TDI lat A'): peak velocity of diastolic mitral annular motion determined by pulsed-wave tissue Doppler imaging at the level of the left ventricular free wall; (TDI sept A'): peak velocity of diastolic mitral annular motion determined by pulsed-wave tissue Doppler imaging at the level of the interventricular wall; (TDI A'ann): average of TDI lat A' and TDI sep A'; (MR vel): mitral regurgitation velocity; (LA/ Ao ratio): left atrium-to-aortic ratio; (LADmin): left atrial linear dimension at ventricular end-diastole; (LADmid): left atrial linear dimension immediately before the P wave on the ECG; (LADmax): left atrial linear dimension at the ventricular end-systole; (LAVmin): left atrial volume at ventricular end-diastole; (LAVmid): left atrial volume immediately before the P wave on the ECG; (LAVmax): left atrial volume at the ventricular end-systole; (PassEmpD-FS): LA passive function based on diameters; (ActEmpD-FS): LA active function based on diameters; (ComEmpD-FS): LA complete function based on diameters; (PassEmpV-EF): LA passive function based on volumes; (ActEmpV-EF): LA active function based on volumes; (ComEmpV-EF): LA complete function based on volumes; (LV): left ventricle; (FS): fractional shortening; (EF): ejection fraction; (ESVI): end-systolic volume index. Comparison of values at the initial diagnosis and at the time of the second echocardiography (1-6 months after starting pimobendan). Values are expressed as mean and standard deviation. $\left(^{*}\right)$ : Statistically significant $p$ values. 
Pimobendan is a benzimidazole-pyridazinone drug, with inodilator effect (Boyle and Leech, 2012). Pimobendan administration has been shown to be beneficial in dogs with both preclinical and symptomatic MMVDs, decreasing LV size and improving LV systolic and diastolic function (Hesse et al., 2004; Häggström et al., 2008; Roşca et al., 2011; Boswood et al., 2018). Furthermore, a number of studies reported a short-term reduction in the left atrial size following administration of pimobendan in dogs with both preclinical and symptomatic MMVDs (Lombard et al., 2006; Kanno et al., 2007; Boswood et al., 2016, 2018). Finally, pimobendan decreased LA pressure in a dose-dependent manner in dogs with experimentally induced MR (Suzuki et al., 2011) although this could possibly represent an indirect effect of pimobendan on the LA (reduced mitral regurgitant stroke volume and increased left ventricular forward stroke volume associated with its inodilator effect).

To the authors' knowledge, the effect of this drug on LA function in dogs has not been previously evaluated. The effect of pimobendan on LA function in cats, both healthy and with hypertrophic cardiomyopathy, has been recently reported, with conflicting results (Kent, 2011; Oldach et al., 2019). A single oral dose of pimobendan was found to increase LA FS in cats with asymptomatic hypertrophic cardiomyopathy (Oldach et al., 2019). In contrast, in healthy cats, pimobendan was reported to reduce LA end-diastolic size but with minimal effect on LA functional indices, following oral treatment for 7 days (Kent, 2011). A possible explanation for the contrasting results of these studies maybe the different times of pimobendan treatment (single dose versus 7 days), suggesting a maximal short-term effect of pimobendan on LA function which decreases thereafter. This finding could explain the neutral results in this study where the treatment was continued for a minimum of 1 month. Similarly, levosimendan, a calcium sensitizer with similar mechanism of action to pimobendan, has been shown to improve the indices of LA function in people with ischemic and decompensated heart failure after 24 hours of treatment (Duygu et al., 2008), but no assessment on its long-term effect has been reported, to the authors' knowledge.

Based on these previous results and the pharmacological properties of pimobendan, an improvement in LA function and dimension was expected following its administration in dogs with preclinical MMVD, as shown by our preliminary data (Sarcinella et al., 2018). The results of this study show no significant changes in LA function or dimensions over the timescale of $<6$ months even though LV size reduced and LV systolic function improved. These results are interesting and may reflect a balance of potential pimobendanassociated improvement in LA size and function offset by progression of the underlying disease, leading to a lack of significantly identifiable positive or negative changes. For example, LA function and dimensions are expected to worsen with the progression of the disease (Nakamura et al., 2014; Dickson et al., 2017) and so this may explain the lack of significant benefit of pimobendan treatment on atrial function in this study, given the timescale. Furthermore, it is possible that the longer time to reexamination (median of 4 months) when compared with the previous reports (Lombard et al., 2006; Kanno et al., 2007; Boswood et al., 2018), where the LA size was reported to decrease after 1-2 months of treatment with pimobendan, and the variable time interval between the two echocardiographic assessments may have contributed to failure to document reduced LA size with pimobendan treatment reported in this study. Interestingly, in support of this, the initial reduction in LV and LA size associated with pimobendan treatment is no longer evident when dogs were evaluated 180 days after institution of treatment (Ouellet et al., 2009). Furthermore, pimobendan has been reported to reduce radiographic vertebral heart sum (VHS) by 3 months of treatment, but then progressively VHS increases again to slightly greater than the baseline level at 6 months (Woolley et al., 2007). This might indicate that time has an impact of measurable effects of pimobendan on cardiac structure and function; as the left atrial size contributes to radiographic cardiac silhouette size, it is possible that the left atrial size also diminishes and then increases again.

While there was no demonstrable improvement in LA size and function, it is important to note that there was also a lack of negative changes in LA function and dimension which may also support a beneficial effect of pimobendan in delaying the progression of the disease, although a maximum of 6 months interval maybe too a short time interval to expect significant progression of this condition.

In addition, it is possible that the lack of demonstrable improvement in LA size or function may reflect a possible tolerance effect to the action of pimobendan, at least in some of the dogs included in this study. Tachyphylaxis of pimobendan in chronic therapy in dogs has not yet been reported but has been hypothesized in a previous study (Ouellet et al., 2009), and a decrease in pimobendan's vasodilator effect has been described in people after 6 months of treatment based on indirect arterial blood pressure monitoring (Erlemeier et al., 1991).

As well as the LA intrinsic contractility, LA active function is modulated by LA preload and LA afterload, determined by LV end-diastolic pressure (Osuga et al., 2016), and results of the previous studies suggest that the LA active function is enhanced in the early phase of volume overload (Osuga et al., 2016). Therefore, it is possible that the lack of statistically positive effect on LA function reported in this study was due to the vasodilator proprieties of pimobendan causing 
decreased LV afterload. Furthermore, the combination of reduced LV afterload and increased LV systolic function following pimobendan administration results in reduction of regurgitant stroke volume (Kanno et al., 2007) and decreased LA preload. This may be associated with a reduction in Frank-Starling mechanism of the LA myocardium, masking any intrinsic increased atrial myocardial contractility secondary to the direct inotropic effect of pimobendan. Indeed, the ability of pimobendan to decrease LA pressure has already been reported in dogs with experimentally induced MR (Suzuki et al., 2011).

The median time to onset of CHF in this study was 552 days. This is a shorter period when compared with what has been reported in the EPIC study (time to end point 1228 days) (Boswood et al., 2016); however, this may be due to the short time period of this study; the smaller number of dogs included and the fact that the majority of the dogs included were not yet in CHF (59\%) when the study was ended.

In addition to the variable time interval between two echocardiographic assessments, other study limitations must be acknowledged. The sample size of $27 \mathrm{dogs}$ is small, which limited statistical power for identifying differences. This might have affected the results of LA dimensions and function and our failure to confirm our hypothesis of reduced LA size and improved function which we expected with pimobendan treatment.

This is a retrospective study, and therefore carries inherent limitations related to this type of the study. For example, the echocardiographic images used for the statistical analysis were acquired by different operators; however, a single observer obtained all LA measurements for this study, aiming to minimize interobserver variability.

This study also lacks a control group; however, following the presentation of the EPIC study results (Boswood et al., 2016) showing a significant benefit of pimobendan administration in dogs affected by stage B2 MMVD, it would have been unethical to replace this drug of known benefit for this subset of dogs with a placebo. Furthermore, before the EPIC results (Boswood et al., 2016), angiotensin-converting enzyme inhibitor, alone or in combination with pimobendan, was anecdotally used in dogs affected by stage B2 MMVD and clinically relevant LA enlargement (Atkins et al., 2009). For this reason, it was not possible to use an historical control group from dogs assessed at our institution before the EPIC study. Nevertheless, as the LA variables were assessed pre- and postpimobendan for each animal included in the study, each dog was acting as its own control.

No advanced imaging, such as three-dimensional and speckle tracking echo, magnetic resonance imaging, or invasive assessment of LA functional indices, was performed. However, 2D noninvasively echocardiographic assessment of LA phasic function has been validated in both human and veterinary medicine
(Blume et al., 2011; Wesselowski et al., 2014) and has been proven to be feasible and reproducible in dogs affected by MMVD (Höllmer et al., 2013; Wesselowski et al., 2014; Caivano et al., 2016). Moreover, TDI A' velocity has been shown to be useful as an accurate and rapid marker of global LA function and correlates with other traditional parameters of atrial function in people (Lindstro and Wranne, 1999; Blume et al., 2011).

\section{Conclusions}

Results of the present study describe, for the first time, the effect of pimobendan on LA phasic function in dogs affected with preclinical (ACVIM stage B2) MMVD, showing no significant changes of 2D- and TDIderived indices of the left atrial phasic function within 6 months after initiation of oral pimobendan. However, the prospective studies with a larger population are needed to investigate this further.

\section{Conflicts of interest}

The authors declare that they do not have any conflicts of interest to disclose.

\section{Authors' contributions}

FS, JN, EFB, and JDM devised the project. FS carried out the additional echocardiographic measurements and analysis for the left atrial function variables. TWM carried out the statistical analysis. All authors were involved in acquisition of the echocardiographic data from dogs included in the study. FS, JN, and JDM prepared the manuscript and HHG and EFB contributed to the final manuscript.

\section{References}

Atkins, C., Bonagura, J., Ettinger, S., Fox, P.R., Gordon, S., Häggström, J., Hamlin, R., Keene, B., LuisFuentes, V. and Stepien, R. 2009. Guidelines for the diagnosis and treatment of canine chronic valvular heart disease. J. Vet. Intern. Med. 23, 1142-1150.

Baron Toaldo, M., Romito, G., Guglielmini, C., Diana, A., Pelle, N.G., Contiero, B. and Cipone, M. 2018. Prognostic value of echocardiographic indices of left atrial morphology and function in dogs with myxomatous mitral valve disease. J. Vet. Intern. Med. 32, 914-921.

Baron Toaldo, M., Guglielmini, C., Diana, A., Sarcinella, F. and Cipone, M. 2014. Feasibility and reproducibility of echocardiographic assessment of regional left atrial deformation and synchrony by tissue Doppler ultrasonographic imaging in healthy dogs. Am. J. Vet. Res. 75, 59-66.

Benjamin, E., D'Agostino, R., Belanger, A.J., Wolf, P.A. and Levy, D. 1995. Left atrial size and the risk of stroke and death. The Framingham Heart Study. Circulation 92, 835-841.

Blume, G.G., Mcleod, C.J., Barnes, M.E., Seward, J.B., Pellikka, P.A., Bastiansen, P.M. and Tsang, T.S. 2011. Left atrial function: physiology, assessment, and clinical implications. Eur. J. Echocardiogr. 12(6), 421-430. 
Borgarelli, M., Savarino, P., Crosara, S., Santilli, R.A., Chiavegato, D., Poggi, M., Bellino, C., La Rosa, G., Zanatta, R., Haggstrom, J. and Tarducci, A. 2008. Survival characteristics and prognostic variables of dogs with mitral regurgitation attributable to myxomatous valve disease. J. Vet. Intern. Med. 22, $120-128$.

Boswood, A., Häggström, J., Gordon, S.G., Wess, G., Stepien, R.L., Oyama, M.A., Keene, B.W., Bonagura, J., MacDonald, K.A., Patteson, M., Smith, S., Fox, P.R., Sanderson, K., Woolley, R., Szatmári, V., Menaut, P., Church, W.M., O'Sullivan, M.L., Jaudon, J.P., Kresken, J.G., Rush, J., Barrett, K.A., Rosenthal, S.L., Saunders, A.B., Ljungvall, I., Deinert, M., Bomassi, E., Estrada, A.H., Fernandez Del Palacio, M.J., Moise, N.S., Abbott, J.A., Fujii, Y., Spier, A., Luethy, M.W., Santilli, R.A., Uechi, M., Tidholm, A. and Watson, P. 2016. Effect of pimobendan in dogs with preclinical myxomatous mitral valve disease and cardiomegaly: the EPIC study - a randomized clinical trial. J. Vet. Intern. Med. 30(6), 1765-1779.

Boswood, A., Gordon, S.G., Häggström, J., Wess, G., Stepien, R.L., Oyama, M.A., Keene, B.W., Bonagura, J., MacDonald, K.A., Patteson, M., Smith, S., Fox, P.R., Sanderson, K., Woolley, R., Szatmári, V., Menaut, P., Church, W.M., O'Sullivan, M.L., Jaudon, J.P., Kresken, J.G., Rush, J., Barrett, K.A., Rosenthal, S.L., Saunders, A.B., Ljungvall, I., Deinert, M., Bomassi, E., Estrada, A.H., Fernandez Del Palacio, M.J., Moise, N.S., Abbott, J.A., Fujii, Y., Spier, A., Luethy, M.W., Santilli, R.A., Uechi, M., Tidholm, A., Schummer, C. and Watson, P. 2018. Longitudinal analysis of quality of life, clinical, radiographic, echocardiographic, and laboratory variables in dogs with preclinical myxomatous mitral valve disease receiving pimobendan or placebo: the EPIC Study. J. Vet. Intern. Med. 32(1), 72-85.

Boyle, K.L. and Leech, E. 2012. A review of the pharmacology and clinical uses of pimobendan. J. Vet. Emerg. Crit. Care. 22(4), 398-408.

Caivano, D., Rishniw, M., Patata, V., Giorgi, M.E. and Porciello, F. 2016. Left atrial deformation and phasic function determined by 2-dimensional speckle tracking echocardiography in healthy dogs. J. Vet. Cardiol. 18, 146-155.

Cornell, C.C., Kittleson, M.D., Della Torre, P., Häggström, J., Lombard, C.W., Pedersen, H.D., Vollmar, A., and Wey, A. 2004. Allometric scaling of M-mode cardiac measurements in normal adult dogs. J. Vet. Intern. Med. 18, 311-321.

Dickson, D., Caivano, D., Matos, J.N., Summerfield, N. and Rishniw, M. 2017. Two dimensional echocardiographic estimates of left atrial function in healthy dogs and dogs with myxomatous mitral valve disease. J. Vet. Cardiol. 19, 469-479.
Duygu, H., Nalbantgil, S., Ozerkan, F., Zoghi, M., Akilli, A., Erturk, U., Akin, M., Nazli, C. and Ergene, O. 2008. Effects of levosimendan on left atrial functions in patients with ischemic heart failure. Clin. Cardiol. 31(12), 607-613.

Erlemeier, H.H., Kupper, W. and Bleifeld, W. 1991. Comparison of hormonal and haemodynamic changes after long-term oral therapy with pimobendan or enalapril-a double-blind randomized study. Eur. Heart J. 12, 889-899.

Fries, R.C., Gordon, S.G., Saunders, A.B., Miller, M.W., Hariu, C.D. and Schaeffer, D.J. 2019. Quantitative assessment of two- and threedimensional transthoracic and two-dimensional transesophageal echocardiography, computed tomography, and magnetic resonance imaging in normal canine hearts. J. Vet. Cardiol. 21, 79-92.

Häggström, J., Boswood, A., O’Grady, M., Jöns, O., Smith, S., Swift, S., Swift, S., Borgarelli, M., Gavaghan, B., Kresken, J.G., Patteson, M., Ablad, B., Bussadori, C.M., Glaus, T., Kovacević, A., Rapp, M., Santilli, R.A., Tidholm, A., Eriksson, A., Belanger, M.C., Deinert, M., Little, C.J., Kvart, C., French, A., Rønn-Landbo, M., Wess, G., Eggertsdottir, AV., O'Sullivan, M.L., Schneider, M., Lombard, C.W., Dukes-McEwan, J., Willis, R., Louvet, A. and DiFruscia, R. 2008. Effect of pimobendan or benazepril hydrochloride on survival times in dogs with congestive heart failure caused by naturally occurring myxomatous mitral valve disease: the QUEST study. J. Vet. Intern. Med. 22(5), 1124-1135.

Hansson, K., Häggström, J., Kvart, C. and Lord, P. 2002. Left atrial to aortic root indices using two dimensional and M-mode echocardiography in cavalier King Charles spaniels with and without left atrial enlargement. Vet. Radiol. Ultrasound 43, 568-575.

Hauser, T.H., Peters, D.C., Wylie, J.V. and Manning, W.J. 2008. Evaluating the left atrium by magnetic resonance imaging. Europace 10(3), 22-27.

Hesse, B., Schuele, S.U., Thamilasaran, M., Thomas, J. and Rodriguez, L. 2004. A rapid method to quantify left atrial contractile function: Doppler tissue imaging of the mitral annulus during atrial systole. Eur. J. Echocardiogr. 5(1), 86-92.

Höllmer, M., Willesen, J.L., Tolver, A. and Koch, J. 2013. Left atrial volume and phasic function in clinically healthy dogs of 12 different breeds. Vet. J. 197(3), 639-645.

Kanno, N., Kuse, H., Kawasaki, M., Hara, A., Kano, R. and Sasaki, Y. 2007. Effects of pimobendan for mitral valve regurgitation in dogs. J. Vet. Med. Sci. 69(4), 373-377.

Kent, A.M. 2011. Effects of atenolol, ivabradine and pimobendan on left atrial and left atrial appendage function: an echocardiographic study 
in healthy cats. MSc Thesis. Ohio State University, Columbus, $\mathrm{OH}$.

Lindstro, L. and Wranne, B. 1999. Pulsed tissue Doppler evaluation of mitral annulus motion: a new window to assessment of diastolic function. Clin. Physiol. 19(1), 1-10.

Linney, C.J., Dukes-McEwan, J., Stephenson, H.M., López-Alvarez, J. and Fonfara, S. 2014. Left atrial size, atrial function and left ventricular diastolic function in cats with hypertrophic cardiomyopathy. J. Small Anim. Pract. 55, 198-206.

Ljungvall, I. and Häggström, J. 2016. Adult-onset valvular heart disease. In: Ettinger, S.J., Feldman, E.C. Texbook of Veterinary Internal Medicine: Diease of the Dog and Cat. Philadelphia: WB Saunders, pp: 1249-1265.

Lombard, C.W., Jons, O. and Bussadori, C.M. 2006. Clinical efficacy of pimobendan versus benazepril for the treatment of acquired atrioventricular valvular disease in dogs. J. Am. Anim. Hosp. Assoc. 42, 249-261.

Nakamura, K., Osuga, T., Morishita, K., Suzuki, S., Morita, T., Yokoyama, N., Ohta, H., Yamasaki, M. and Takiguchi, M. 2014. Prognostic value of left atrial function in dogs with chronic mitral valvular heart disease. J. Vet. Intern. Med. 28, 1746-1752.

Oldach, M.S., Ueda, Y., Ontiveros, E.S., Fousse, S.L., Harris, S.P. and Stern, J.A. 2019. Cardiac effects of a single dose of pimobendan in cats with hypertrophic cardiomyopathy; a randomized, placebo-controlled, crossover study. Front. Vet. Sci. $6,15$.

Osuga, T., Nakamura, K., Morita, T., Nisa K., Sasaki N., Morishita K., Ohta H. and Takiguchi M. 2016. Effects of experimental cardiac volume loading on left atrial phasic function in healthy dogs. Am. J. Vet. Res. 77, 952-960.

Ouellet, M., Bélanger, M.C., Difruscia, R. and Beauchamp, G. 2009. Effect of Pimobendan on Echocardiographic Values in Dogs with Asymptomatic Mitral Valve Disease. J. Vet. Emerg. Crit. Care 23(2), 258-263.

Pritchett, A.M., Jacobsen, S.J., Mahoney, D.W., Rodeheffer, R.J., Bailey, K.R. and Redfield, M.M. 2003. Left Atrial volume as an index of left atrial size: a population-based study. J. Am. Coll. Cardiol. 41, 1036-1043.

Rishniw, M. and Erb, H.N. 2000. Evaluation of four 2-dimensional echocardiographic methods of assessing left atrial size in dogs. J. Vet. Intern. Med. $14,429-435$.

Roşca, M., Lancellotti, P., Popescu, B.A. and Piérard, L.A. 2011. Left atrial function: pathophysiology, echocardiographic assessment, and clinical applications. Heart 97, 1982-1989.
Russo, C., Hahn, R.T., Jin, Z., Homma, S., Sacco, RL. and Di Tullio, M.R. 2010. Comparison of echocardiographic single-plane versus biplane method in the assessment of left atrial volume and validation by real time three-dimensional echocardiography. J. Am. Soc. Echocardiogr. 23(9), 954-960.

Sahn, D.J., DeMaria, A., Kisslo, J. and Weyman, A. 1978. Recommendations regarding quantitation in M-mode echocardiography: results of a survey of echocardiographic measurements. Circulation 58, 1072-1083.

Sarcinella, F., Neves, J., Bode, L., Hodgkiss-Geere, H. and Dukes-McEwan, J. 2018. Effect of pimobendan on left atrial function in dogs with stage B2 myxomatous mitral valve disease. Available via ECVIM. https://www.vin.com/doc/?id=8623295).

Strohm, L.E., Visser, L.C., Chapel, E.H., Drost, W.T. and Bonagura, J.D. 2018. Two-dimensional, long axis echocardiographic ratios for assessment of left atrial and ventricular size in dogs. J. Vet. Cardiol. 20(5), 330-342.

Suzuki, S., Fukushima, R., Ishikawa, T., Hamabe, L., Aytemiz, D. and Huai-Che, H. 2011. The effect of pimobendan on left atrial pressure in dogs with mitral valve regurgitation. J. Vet. Intern. Med. 25(6), 1328-1333.

Thomas, W.P., Gaber, C.E., Jacobs, G.J., Kaplan, P.M., Lombard, C.W., Moise, N.S. and Moses, B.L. 1993. Recommendations for standards in transthoracic two-dimensional echocardiography in the dog and cat. Echocardiography Committee of the Specialty of Cardiology, American College of Veterinary Internal Medicine. J. Vet. Intern. Med. 7, 247-252.

Tidholm, A., Höglund, K., Häggström, J., BodegårdWestling, A. and Ljungvall, I. 2013. Left atrial ejection fraction assessed by real-time 3-dimensional echocardiography in normal dogs and dogs with myxomatous mitral valve disease. J. Vet. Intern. Med. 27(4), 884-889.

Verheule, S., Wilson, E., Everett, T., Shanbhag, S., Golden, C. and Olgin, J. 2003. Alterations in atrial electrophysiology and tissue structure in a canine model of chronic atrial dilatation due to mitral regurgitation. Circulation 107, 2615-2622.

Wess, G., Domenech, O., Dukes-McEwan, J., Häggström, J. and Gordon, S. 2017. European Society of Veterinary Cardiology screening guidelines for dilated cardiomyopathy in Doberman Pinschers. J. Vet. Cardiol. 19(5), 405-415.

Wess, G., Maürer, J., Simak, J. and Hartmann, K. 2010. Use of Simpson's method of disc to detec early echocardiographic changes in Doberman Pinschers with dilated cardiomyopathy. J. Vet. Intern. Med. 24, 1069-1076. 
Wesselowski, S., Borgarelli, M., Bello, N.M. and Abbott, J. 2014. Discrepancies in identification of left atrial enlargement using left atrial volume versus left atrial-to-aortic root ratio in dogs. J. Vet. Intern. Med. 28(5), 1527-1533.
Woolley, R., Smith, P., Munro, E., Smith, S., Swift, S., Devine, C., Corcoran B. and French A. 2007. Effects of treatment type on vertebral heart size in dogs with myxomatous mitral valve disease. Int. J. Appl. Res. Vet. Med. 5(1), 43-48. 